\title{
Regulation of tumor-associated macrophage (TAM) differentiation by NDRG 2 expression in breast cancer cells
}

\author{
Soyeon Lee \\ Department of Biological Science and the Cellular Heterogeneity Research Center, Sookmyung Women's University, Seoul 04310, Korea
}

\begin{abstract}
Macrophages are a major cellular component of innate immunity and are mainly known to have phagocytic activity. In the tumor microenvironment (TME), they can be differentiated into tumorassociated macrophages (TAMs). As the most abundant immune cells in the TME, TAMs promote tumor progression by enhancing angiogenesis, suppressing $T$ cells and increasing immunosuppressive cytokine production. $\mathrm{N}$-myc downstream-regulated gene 2 (NDRG2) is a tumor suppressor gene, whose expression is downregulated in various cancers. However, the effect of NDRG2 on the differentiation of macrophages into TAMs in breast cancer remains elusive. In this study, we investigated the effect of NDRG2 expression in breast cancer cells on the differentiation of macrophages into TAMs. Compared to tumor cell-conditioned medium (TCCM) from 4T1-mock cells, TCCM from NDRG2-overexpressing 4T1 mouse breast cancer cells did not significantly change the morphology of RAW 264.7 cells. However, TCCM from 4T1-NDRG2 cells reduced the mRNA levels of TAM-related genes, including MR1, IL-10, ARG1 and iNOS, in RAW 264.7 cells. In addition, TCCM from 4T1-NDRG2 cells reduced the expression of TAM-related surface markers, such as CD206, in peritoneal macrophages (PEM). The mRNA expression of TAMrelated genes, including IL-10, YM1, FIZZ1, MR1, ARG1 and iNOS, was also downregulated by TCCM from 4T1-NDRG2 cells. Remarkably, TCCM from 4T1-NDRG2 cells reduced the expression of PD-L1 and Fra-1 as well as the production of GM-CSF, IL-10 and ROS, leading to the attenuation of T cellinhibitory activity of PEM. These data showed that compared with TCCM from 4T1-mock cells, TCCM from 4T1-NDRG2 cells suppressed the TAM differentiation and activation. Collectively, these results suggest that NDRG2 expression in breast cancer may reduce the differentiation of macrophages into TAMs in the TME. [BMB Reports 2022; 55(2): 81-86]
\end{abstract}

*Corresponding author. Tel: +82-2-710-9560; Fax: +82-2-2077-7322; E-mail: jslim@sookmyung.ac.kr

${ }^{\#}$ These authors contributed equally to this work.

https://doi.org/10.5483/BMBRep.2022.55.2.114

Received 13 August 2021, Revised 8 September 2021, Accepted 26 October 2021

Keywords: Breast cancer, Macrophage differentiation, NDRG2, Tumorassociated macrophages, Tumor microenvironment

\section{INTRODUCTION}

Macrophages are major innate immune cells and can be found in almost every organ. Macrophages are mainly known as phagocytic cells and can be divided into two major subsets: classically activated macrophages (M1 macrophages), which have pro-inflammatory and tumor-suppressive activity, and alternatively activated macrophages (M2 macrophages), which have antiinflammatory and tumor-promoting activity.

The environment around a tumor is the tumor microenvironment (TME). The TME contains immune cells, extracellular matrix (ECM), tumor cells and signaling molecules. The TME profoundly affects tumor progression and immunosuppression via extracellular paracrine signals. Macrophages are one of the abundant immune cells in the TME and are recruited into tumors by various soluble factors, including vascular endothelial growth factor (VEGF) and C-C chemokine ligand 2 (CCL2) $(1,2)$. They also differentiate into tumor-associated macrophages (TAMs) via their interaction with tumor and tumor-derived factors. In humans, TAMs are defined as CD14 ${ }^{\text {hi }} \mathrm{CD} 11 \mathrm{~b}^{+} \mathrm{HLA}^{-D R^{+}} \mathrm{CD} 163^{+}$ cells. In mice, TAMs express CD11b, F4/80, CD206 and CD163 $(3,4)$. Previous studies have reported that YM1, FIZZ1 and MR1 (CD206) are TAM differentiation markers and that ARG1, iNOS, PD-L1 and PD-1 are TAM activation markers (5-9).

TAMs are similar to M2 macrophages and promote tumor progression via suppression of $\mathrm{T}$ cells, generation of immune suppressive cytokines and induction of angiogenesis. In particular, TAMs are well known to suppress immune responses. First, interleukin-10 (IL-10) and transforming growth factor beta (TGF- $\beta$ ) produced by TAMs induce the generation of regulatory $\mathrm{T}$ (Treg) cells, suppression of T cells and inhibition of dendritic cell (DC) maturation. TAMs induce metabolic starvation of $\mathrm{T}$ cells via secretion of indoleamine 2,3-dioxygenase 1/2 (IDO1/2) and arginase 1 (ARG1). Importantly, they can also suppress the anti-tumor $\mathrm{T}$ cell responses through the expression of PD-L1/2 $(4,10)$. As various types of anti-cancer therapies have side effects, preclinical and clinical studies are focused on immunotherapies that cause fewer side effects than other anti-cancer therapies. However, macrophages recruited in response to immunotherapies tend to differentiate into TAMs in the TME, which is a limitation of these therapies. For this reason, targeting TAMs has emerged as a novel strategy for anti-cancer therapies against solid tumors (4).

The N-myc downstream-regulated gene (NDRG) family consists 
of NDRG1-4. NDRG family proteins are characterized by an $\alpha / \beta$ hydrolase fold of approximately 220 amino acids and a lipase/esterase/thioesterase active site $(11,12)$. NDRG2 is known to be a cell stress-related and tumor suppressor gene in multiple solid tumors, including breast cancer $(13,14)$, colorectal cancer (15), renal cell carcinoma (16) and lung cancer (17). NDRG2 expression can inhibit breast cancer development by decreasing tumor cell proliferation, migration and epithelial-mesenchymal transition (EMT) $(13,14)$.

Our previous study revealed that NDRG2 overexpression suppresses osteoclast differentiation via the inhibition of ICAM-1 expression in breast cancer (18). This suggests that NDRG2 overexpression may control the differentiation of other immune cells. However, the effect of NDRG2 expression in breast cancer on the differentiation of macrophages into TAMs has not been investigated. Thus, in the present study, we aimed to examine the effect of NDRG2-overexpressing breast cancer cells on the differentiation of macrophages into TAMs.

\section{RESULTS}

\section{Evaluation of NDRG2 expression levels in 4T1-NDRG2 cells} prior to TCCM generation

To evaluate whether NDRG2 expression in breast cancer cells affects the differentiation of macrophages into TAMs, tumor cell-conditioned medium (TCCM), which are known to mimic the TME, were collected from $4 \mathrm{~T} 1$ cells. The $4 \mathrm{~T} 1$ cell line, a mouse mammary carcinoma cell line, was derived from Balb/c mice and has been characterized as a highly metastatic tumor model. 4T1 cells were stably transfected with the pCMV/Tag2Bempty vector (mock) or pCMV/Tag2B-NDRG2 vector. NDRG2 mRNA and protein were confirmed to be expressed at the expected levels by RT-PCR and western blot analysis (Fig. 1A). Difference in cell viability in both cell lines was not observed (Supplementary Fig. 1). In addition, the production of GM-CSF and IL-10 associated with immune suppression in $4 \mathrm{~T} 1$ cell was significantly decreased by the transfection with NDRG2 (Fig. 1B).

\section{TCCM from NDRG2-overexpressing 4T1 cells reduced TAM differentiation and activation of RAW 264.7 cells}

To differentiate macrophages into TAMs, the culture media of 4T1-mock and 4T1-NDRG2 cells were obtained and concentrated. It was previously reported that when macrophages are treated with TCCM from 4T1 cells to induce TAM differentiation, they become elongated (19). RAW 264.7 cells, which are mouse macrophages, were derived from Balb/c mice and are usually used for studies on macrophage differentiation (20). TCCM from 4T1-NDRG2 cells (4T1-N2 CM) and TCCM from 4T1-mock cells (4T1-M CM) altered the elongated morphology of RAW 264.7 cells (Fig. 1C). Since there was little difference in the morphological change in RAW 264.7 cells after treatment with 4T1-N2 CM or 4T1-M CM, the expression of TAM differentiation and activation markers was assessed by RT-PCR (Supplementary Fig. 2) and RT-qPCR (Fig. 1D). The expression

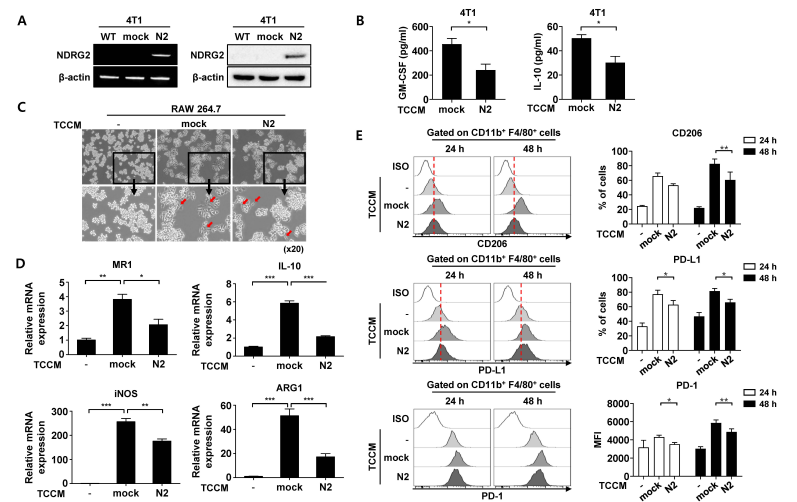

Fig. 1. The expression of TAM differentiation and activation markers in RAW 264.7 cells was decreased by 4T1-N2 CM. (A) The mRNA and protein levels of NDRG2 in 4T1 cells were measured by RT-PCR and western blot analysis. (B) The 4T1-mock and 4T1-NDRG2 cells were cultured for $48 \mathrm{~h}$, and then IL-10 and GM-CSF levels in the supernatants were determined by sandwich ELISA kits. (C) To generate TAMs, RAW 264.7 cells were treated with 4T1-M CM or 4T1-N2 $\mathrm{CM}$ for $24 \mathrm{~h}$. Cell morphology images were captured using a microscope $(\times 20)$. (D) The mRNA expression levels of TAM differentiation markers (MR1) and activation markers (IL-10, iNOS and ARG1) were measured by quantitative real-time PCR. (E) The CD206, PD-L1 and PD-1 expression in TAM was quantified by flow cytometry. The results are presented as the mean \pm SEM. The data are representative of at least three independent experiments. ${ }^{*} \mathrm{P}<0.05,{ }^{*} \mathrm{P}<0.01$, $* * * P<0.001$

of TAM differentiation marker (MR1) and TAM activation markers (IL-10, ARG1 and iNOS) was reduced in cells treated with 4T1-N2 CM compared with those treated with 4T1-M CM. Furthermore, the surface expression of CD206, PD-L1 and PD-1 was also suppressed after treatment with 4T1-N2 CM (Fig. 1E). These results suggested that TAM differentiation and activation were attenuated in RAW 264.7 cells treated with 4T1-N2 CM compared with those treated with 4T1-M CM.

4T1-N2 CM attenuated TAM differentiation and activation in peritoneal macrophages

Peritoneal macrophages (PEMs) have been widely used for studies of macrophage polarization and TAM differentiation (21-23). To examine the effect of NDRG2 expression in 4T1 cells on the differentiation of macrophages into TAMs, murine PEMs were isolated from Balb/c mice. As shown in Fig. 2A, more than $85 \%$ of PEMs isolated from the murine peritoneal cavity were F4/80-positive. 4T1-N2 CM slightly altered the elongated morphology of PEMs induced by treatment with 4T1-M CM (Fig. 2B). To compare the effect of 4T1-M CM and 4T1-N2 $\mathrm{CM}$ on the induction of TAMs, the expression of TAM differentiation and activation markers was analyzed. TAMs usually exhibit the M2 phenotype, and CD206 has been reported to be a TAM differentiation markers. In addition, PD-L1 and PD-1 have been reported to be TAM activation markers $(8,9)$. Interestingly, compared with 4T1-M CM, 4T1-N2 CM significantly 


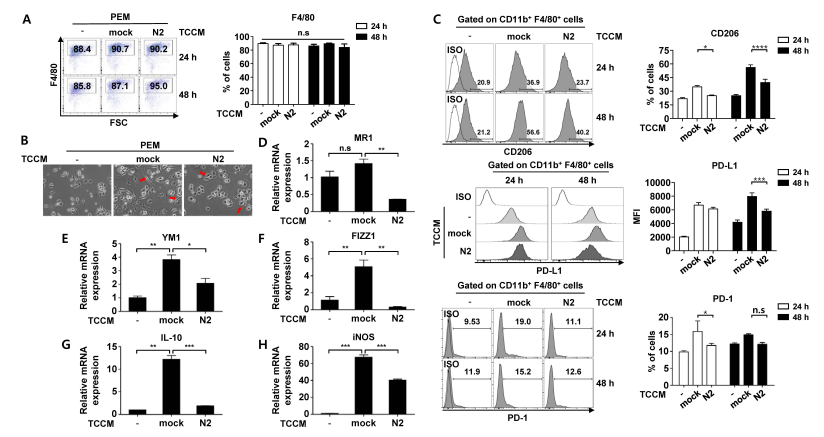

Fig. 2. Compared with 4T1-M CM, 4T1-N2 CM inhibited TAM differentiation and activation in PEMs. (A) To confirm the purity of the obtained PEMs, the cells were stained with anti-F4/80. The purity of PEMs was analyzed by flow cytometry. (B) PEMs were seeded in a 6-well plate $\left(8 \times 10^{5}\right)$ and treated with 4T1-M CM or 4T1-N2 CM for $48 \mathrm{~h}$. Cell morphology images were captured using a microscope $(\times 20)$. (C) The cells were stained with specific antibodies aganist CD11b, F4/80, CD206, PD-1 and PD-L1 for cell analysis. The expression levels of TAM differentiation marker (CD206) and TAM activation markers (PD-L1 and PD-1) were measured by flow cytometry, and these data were analyzed using Flowjo software. (D-H) The mRNA expression levels of TAM differentiation markers (MR1, YM1 and FIZZ1) and activation markers (IL-10 and iNOS) were measured by quantitative real-time PCR. The results are presented as the mean \pm SEM. The data are representative of at least three independent experiments. $* \mathrm{P}<0.05, * * \mathrm{P}<0.01, * * * \mathrm{P}<0.001, * * * * \mathrm{P}<0.0001$.

reduced the expression levels of CD206, PD-L1 and PD-1 on PEMs (Fig. 2C). 4T1-M CM treatment enhanced the mRNA expression of TAM differentiation (MR1, YM1 and FIZZ1) and activation (IL-10 and ARG1) markers (Supplementary Fig. 3). Consistent with the results obtained with RAW 264.7 cells, the mRNA expression level of MR1, TM1, FIZZ1, iNOS and IL-10 was also reduced after treatment with 4T1-N2 CM compared with 4T1-M CM (Fig. 2D-H). These data suggested that compared with 4T1-M CM, 4T1-N2 CM had a diminished ability to induce TAM differentiation and activation.

\section{The differential effects of 4T1-M CM and 4T1-N2 CM on the differentiation of macrophages into TAMs}

Next, to assess whether the two kinds of $\mathrm{CM}$ have similar abilities to induce the differentiation of macrophages into TAMs, the expression levels of some common markers were investigated. TGF- $\beta$ and MMP-9 expressed by TAMs are known to contribute to the immunosuppressive response and tumor metastasis (4). Fra-1 expressed by TAMs is also known to promote the generation of TAMs by binding to the IL-6 promoter (24). Consistent with previous data, treatment of RAW 264.7 cells with 4T1-N2 CM attenuated the mRNA expression of Fra-1 and IL-6 compared with 4T1-M CM (Fig. 3A, B). On the other hand, both types of CM similarly increased the mRNA expression level of TGF- $\beta$ (Fig. $3 \mathrm{C}$ ). The protein expression of Fra-1 and PD-L1 was decreased by 4T1-N2 CM, whereas the expression of MMP-9 was increased by the two types of CM to a similar degree (Fig. 3D). Similar results were obtained in

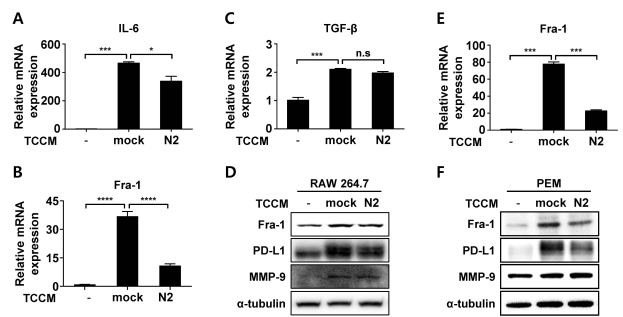

Fig. 3. 4T1-M CM and 4T1-N2 CM differentially increased the expression of other TAM activation markers. RAW 264.7 or PEM cells were treated with 4T1-M CM or 4T1-N2 CM for $24 \mathrm{~h}$. (A-C) The mRNA expression levels of TAM activation markers (IL-6, Fra- 1 and TGF- $\beta$ ) in RAW 264.7 cells were measured by quantitative real-time PCR. (D and F) The protein levels of TAM activation markers (Fra-1, PD-L1 and MMP-9) were measured by western blot analysis. (E) The mRNA expression level of Fra- 1 in PEM was measured by quantitative real-time PCR. The results are presented as the mean \pm SEM. The data are representative of at least three independent experiments. $* P<0.05, * * * P<0.001, * * * * P<0.0001$.
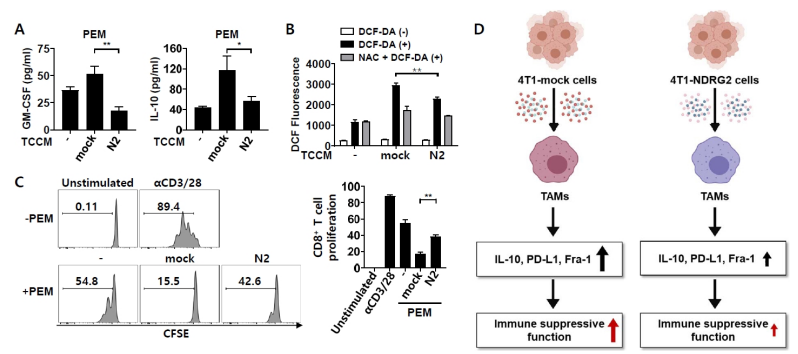

Fig. 4. Comparative analysis of immunosuppressive effects of PEM treated with 4T1-M CM or 4T1-N2 CM. (A) PEMs were treated with $4 \mathrm{~T} 1-\mathrm{M}$ or $-\mathrm{N} 2 \mathrm{CM}$ for $48 \mathrm{~h}$, and then cultured in serum-free medium. After $48 \mathrm{~h}$, the supernatant was collected for ELISA. (B) The cells pretreated in the absence or presence of $10 \mathrm{mM} \mathrm{NAC}$ were cultured with $4 \mathrm{~T} 1-\mathrm{M}$ or $-\mathrm{N} 2 \mathrm{CM}$ for $24 \mathrm{~h}$ and were treated with $10 \mu \mathrm{M}$ DCF-DA for $30 \mathrm{~min}$. Harvested cells were analyzed by flow cytometry. (C) In vitro generated PEM was co-cultured with CFSE-labeled $\mathrm{CD}^{+} \mathrm{T}$ cells at $1: 2$ (PEM:T) ratio for $72 \mathrm{~h}$. The $\mathrm{CD}^{+} \mathrm{T}$ cell proliferation was measured by flow cytometry. $* P<0.05, * * P<0.01$. (D) Model demonstrating the effect of NDRG2 expression in breast cancer on TAM activation. NDRG2 expression in breast cancer cells reduces the expression levels of IL-10, PD-L1 and Fra-1 in TAMs and attenuates the immunosuppressive function of TAMs.

experiments using PEM (Fig. 3E, F). These results suggested that, as revealed by downregulation of IL-6, Fra-1 and PD-L1 expression, 4T1-N2 CM increased TAM differentiation and activation to a less extent than $4 \mathrm{~T} 1-\mathrm{M} \mathrm{CM}$ through downregulation of IL-10, PD-L1 and Fra-1 expression.

\section{T1-N2 CM induced significantly less activation in peritoneal} macrophages than 4T1-M CM

To investigate whether TAM activation in PEM is associated with the production of immune suppressive factors, GM-CSF, IL-10 and reactive oxygen species (ROS) levels were measured 
in PEM after treatment with TCCM. Compared with 4T1-M CM, 4T1-N2 CM induced low level of GM-CSF, IL-10 and ROS production in PEM (Fig. 4A, B). Finally, we assessed the T cell suppressive effect of TCCM-treated PEM by co-culturing with CFSE-labeled mouse $\mathrm{CD}^{+} \mathrm{T}$ cells. As shown in Fig. 4C, 4T1-M CM-pretreated PEM markedly suppressed the proliferation of $\mathrm{CD}^{+}{ }^{+}$cells in the presence of anti-CD3/CD28 antibodies compared with untreated PEM, whereas T cell proliferation was weakly inhibited by a treatment of PEM with 4T1-N2 CM. These findings showed that although 4T1-M CM could induce PEM to strongly suppress $T$ cell proliferation, the suppressive effect was diminished greatly by $4 \mathrm{~T} 1-\mathrm{N} 2 \mathrm{CM}$.

\section{DISCUSSION}

Macrophages have a pivotal role in tumor progression and differentiation by the tumor microenvironment. TAMs in the TME can promote tumor growth and support an immunosuppressive TME. TAMs are similar to M2 macrophages, which promote tumor initiation, progression and metastasis. Preclinical and clinical studies have demonstrated that TAM accumulation in tumors correlates with a poor prognosis $(10,25,26)$. Hence, targeting TAMs could emerge as a novel strategy for cancer therapy. Given that macrophages are required for the efficacy of cancer immunotherapy, depleting TAMs during cancer therapy may impair the efficacy of immunotherapies. Therefore, instead of depleting them, attempts have been made to repolarize macrophage phenotypes.

NDRG2 expression is decreased in various solid tumors, including breast cancer, colorectal cancer and lung cancer. In addition, NDRG2 inhibits metastasis, angiogenesis and invasion $(14,15,17)$. A previous study reported that NDRG2 overexpression in breast cancer suppresses osteoclast differentiation by downregulating ICAM expression (18), suggesting that NDRG2 expression can indirectly modulate the differentiation of other immune cells. However, it has not been reported whether NDRG2 is strongly associated with the differentiation of TAMs in breast cancer. Thus, in this study, we sought to investigate whether NDRG2 expression in breast cancer cells is able to regulate the differentiation of TAMs. In the present study, we demonstrated that compared with 4T1-M CM, 4T1-N2 CM significantly reduced TAM differentiation and activation as revealed by the reduction in expression levels of TAM differentiation and activation markers. While the expression of differentiation markers was relatively weak, the difference in the expression of activation markers was very noticeable (Fig. 2C). It has already been reported that monocyte-derived TAMs can be generated reliably using tumor cell-conditioned medium plus a cytokine cocktail of IL-4, IL-10 and M-CSF (27). In fact, we demonstrated that compared to control vector-transfected cells, NDRG2transfected monocytes and human histiocytic leukemia U937 cells exhibit significantly decreased IL-10 production (28), supporting the hypothesis that decreased tumor-derived IL-10 production after NDRG2 transfection might play a role in reducing the TAM differentiation of macrophages.

In a previous report, Fra-1 expression increased the production of IL-6 in macrophages by binding to the IL- 6 promoter. IL-6 expression induced by Fra- 1 can lead to the differentiation of macrophages into TAMs (24). IL-6 also enhances tumor progression by activating EMT and increases tumor resistance to apoptosis (29). Moreover, IL-6 provokes resistance to chemotherapy (30), and high IL-6 expression correlates with poor prognosis in breast cancer (31). Several lines of evidence suggest that PD-L1 and PD-1 expressed on TAMs also contribute to the immunosuppressive function of TAMs by inactivating T cells and inhibiting anti-tumor immunity $(4,8,9)$. TGF- $\beta$ secreted by TAMs promotes tumor metastasis by upregulating the expression of SRY-related high mobility group box 9 (SOX9) (32). MMP-9 secreted by TAMs promotes tumor metastasis via activation of the PI3K/AKT signaling pathway and induces tumor cell invasion $(25,33)$. Although there was no significant difference in the expression of TGF- $\beta$ and MMP-9 between 4T1-M CM-treated and 4T1-N2 CM-treated macrophages, compared with 4T1-M CM, 4T1-N2 CM substantially reduced the expression of IL-10, Fra-1 and PD-L1 in macrophages (Fig. 3). These results suggested that compared with 4T1-M CM, 4T1-N2 CM reduced the immunosuppressive function of TAMs. However, further study is required to thoroughly investigate the mechanism by which 4T1-N2 CM diminish TAM activation.

In summary, we identified in the present study that NDRG2 expression in breast cancer cells inhibits the expression of IL-10, Fra-1 and PD-L1 in TAMs. Downregulation of IL-10, Fra-1 and PD-L1 expression is expected to reduce the immunosuppressive function of TAMs (Fig. 4D). Consequently, TAM activation at the tumor site surrounded by NDRG2-overexpressing breast cancer cells is reduced, leading to the downregulation of IL-10, Fra-1 and PD-L1 expression. These findings suggest that NDRG2 could be a novel regulatory factor that modulates TAM activation in cancer therapy.

\section{MATERIALS AND METHODS}

\section{Cell culture and reagents}

Mouse breast cancer cell lines (4T1-mock and 4T1-NDRG2) and a mouse macrophage cell line (RAW 264.7) were cultured in DMEM (Gibco/Invitrogen, Carlsbad, CA) containing 1\% penicillin/streptomycin and $10 \%$ heat-inactivated fetal bovine serum (FBS) (Gibco/Invitrogen) at $37^{\circ} \mathrm{C}$ in a humidified $95 \%$ air $/ 5 \%$ $\mathrm{CO}_{2}$ incubator.

\section{Tumor cell-conditioned medium (TCCM)}

4T1-mock and 4T1-NDRG2 cells were cultured in DMEM containing $3 \%$ heat-inactivated FBS for $48 \mathrm{~h}$ and the cell supernatants were collected when the cell densities were $3 \times$ $10^{6}$ cells/plate. The supernatants were concentrated at 3,000 $\times$ g for 30 min at $4^{\circ} \mathrm{C}$ using Amicon Ultra-15 centrifugal filter 3K devices (Merck Millipore, Billerica, MA). The concentrated media were diluted with DMEM containing 3\% FBS (Gibco/ 
Invitrogen) to produce $30 \%$ concentrated media.

\section{RNA isolation, quantitative real-time reverse transcription-polymerase chain reaction and RT-PCR}

All cells were lysed using TRIzol reagent (Molecular Research Center Inc, Cincinnati, $\mathrm{OH}$ ), and total RNA was isolated according to the manufacturer's instructions. Total RNA (5 $\mu \mathrm{g})$ was reverse transcribed into cDNA. For the reverse transcription (RT) reaction, first-strand CDNA was generated using M-MLV reverse transcriptase (Promega, Madison, Wl), oligo-(dT) primers and dNTPs (Bioneer, Daejeon, Republic of Korea). The cDNAs were amplified with $10 \mathrm{mM}$ dNTPs, $0.5 \mathrm{U}$ of Taq DNA polymerase and 10 pmol each primer (Bioneer). The PCR products were electrophoresed on $1 \%$ agarose gels containing ethidium bromide. Quantitative real-time PCR was performed using an ABI StepOnePlus ${ }^{T M}$ real-time PCR thermal cycler with Power SYBR Green PCR Master Mix. The experiments were performed in triplicate. The primer sequences for quantitative real-time PCR and RT-PCR are listed in the Supplementary Table 1.

\section{Isolation of peritoneal macrophages}

Female Balb/c mice at 6 to 8 weeks of age were purchase from Daehan Bio link (Eumseong, Republic of Korea). The mice were maintained in specific pathogen-free facilities at Sookmyung Women's University. The animal experiments were performed in accordance with the guidelines of the Institutional Animal Care and Use Committee after approval by the Institutional Ethical Committee of Sookmyung Women's University (Resolution No. SMWU-IACUC-1912-024-1). The method used to isolate peritoneal macrophages from mice was described previously (34). In order to isolate peritoneal macrophages, Balb/c mice were sacrificed, and $10 \mathrm{ml}$ of cold RPMI (Gibco/Invitrogen) medium was injected into the abdominal cavity. The medium was withdrawn and centrifuged $\left(1,500 \mathrm{rpm}\right.$ for $5 \mathrm{~min}$ at $\left.4^{\circ} \mathrm{C}\right)$, and the cell pellet was resuspended in $1 \mathrm{ml}$ of RPMI medium. These peritoneal exudate cells were cultured in dishes $(>4 \mathrm{~h}$ at $37^{\circ} \mathrm{C}$ ). After $2 \mathrm{~h}$, the non-adherent cells were removed.

\section{Western blot analysis and enzyme-linked immunosorbent assay (ELISA)}

All cells were lysed in protein extraction solution (iNtRON Biotechnology, Seongnam, Republic of Korea) The proteins were mixed with $5 \mathrm{X}$ sample buffer and separated by electrophoresis on gradient SDS-PAGE gels ( $8 \%$ to $15 \%$ ). Then, the proteins were transferred onto PVDF membranes (Amersham Biosciences, Buckinghamshire, UK). The membranes were blocked with Tris-buffered saline plus $0.05 \%$ Tween-20 (TBST) with 5\% skim milk for $30 \mathrm{~min}$ and incubated with primary antibodies overnight at $4^{\circ} \mathrm{C}$. After incubation, the membranes were washed and incubated with HRP-conjugated secondary antibodies. The blots were visualized with an enhanced chemiluminescence system using an Ez-Capture MG system (ATTO Corporation, Tokyo, Japan). Specific antibodies against NDRG2, $\beta$-actin and Fra-1 were purchased from Santa Cruz Biotechnology (Santa
Cruz, CA). PD-L1, $\alpha$-tubulin and MMP-9 antibodies were purchased from Cell Signaling Technology Inc. (Beverly, MA). For the measurement of IL-10 (Cat No: 900-TM53) and GM-CSF (Cat No: 900-M55) levels, the culture supernatants were collected and sandwich ELISA kits (Peprotech Inc., Rocky Hill, NJ) were used according to the manufacturer's protocol.

\section{Flow cytometry}

The cell surfaces was stained with antibodies at $4^{\circ} \mathrm{C}$ for 30 min. Specific antibodies against CD11b (M1/70), F4/80 (BM8), CD206 (MMR), PD-1 (J43) and PD-L1 (MIH5) were purchased from eBioscience (San Diego, CA). The samples were washed using PBS and fixed with $4 \%$ paraformaldehyde. The samples were analyzed using FACSCanto $\Pi^{\mathrm{TM}}$ flow cytometer (BD Biosciences, San Jose, CA). The data were analyzed using Flowjo software (Tree Star, Ashland, OR). Cell apoptosis was measured using a double staining with FITC-labeled Annexin V/Propidium lodide (PI) Apoptosis Detection Kit (BD Biosciences) according to the manufacturer's instruction. The apoptosis levels were examined by flow cytometry. For reactive oxygen species (ROS) measurement, the cells were pretreated with $10 \mathrm{mM} \mathrm{NAC}$ (Sigma-Aldrich, St. Louis, MO) for 24 h. Then, $10 \mu \mathrm{M}$ DCF-DA (Sigma-Aldrich) was added to the cells for $30 \mathrm{~min}$ at $37^{\circ} \mathrm{C}$, and DCF fluorescence was measured by flow cytometry.

\section{In vitro $\mathrm{T}$ cell proliferation assay}

Mouse $\mathrm{CD}^{+}{ }^{+}$T cells were isolated from spleens using a S3 ${ }^{\mathrm{TM}}$ Cell Sorter (Bio-Rad Laboratories, Hercules, CA). Sorted CD3 ${ }^{+}$ $\mathrm{T}$ cells with the high purity $(>95 \%)$ using an antibody (145$2 \mathrm{C} 11$, eBioscience) were labeled with carboxyfluorescein succinimidyl ester (CFSE) $(2.5 \mu \mathrm{M}$; Invitrogen) according to the manufacturer's guideline and plated at $1 \times 10^{5} /$ well in 96-well plates containing $2 \mu \mathrm{g} / \mathrm{ml}$ anti-CD3 (145-2C11, eBioscience) and $2 \mu \mathrm{g} / \mathrm{ml}$ anti-CD28 (37.51, eBioscience) mAbs. After 3 days, the cells were stained with CD8 antibody (53-6.7, eBioscience), and then the $\mathrm{CD}^{+} \mathrm{T}$ cell proliferation was determined by flow cytometry at the PEC/T cell ratio of $1: 2$.

\section{Statistical analysis}

Statistical significance was analyzed by one-way ANOVA (Tukey's post hoc test for multiple comparisons) with GraphPad Prism software version 9 (GraphPad Software, San Diego, CA). The values are presented at the mean \pm SEM. $\mathrm{P}<0.05$ was considered significant.

\section{ACKNOWLEDGEMENTS}

This study was supported by grants from the National Research Foundation of Korea (NRF) funded by the Ministry of Science and ICT, Republic of Korea (NRF-2016R1A5A1011974 and 2019 R1A2C1088825). J.L. was supported by a grant from the National Research Foundation of Korea (NRF-2019R1I1A1A01058629). 


\section{CONFLICTS OF INTEREST}

The authors have no conflicting interests.

\section{REFERENCES}

1. Riabov V, Gudima A, Wang N, Mickley A, Orekhov A and Kzhyshkowska J (2014) Role of tumor associated macrophages in tumor angiogenesis and lymphangiogenesis. Front Physiol 5,75

2. Qian B-Z, Li J, Zhang H et al (2011) CCL2 recruits inflammatory monocytes to facilitate breast-tumour metastasis. Nature 475, 222-225

3. Cassetta L, Noy R, Swierczak A et al (2016) Isolation of mouse and human tumor-associated macrophages. Adv Exp Med Biol 899, 211-229

4. Chen Y, Song Y, Du W, Gong L, Chang H and Zou Z (2019) Tumor-associated macrophages: an accomplice in solid tumor progression. J Biomed Sci 26, 78

5. Brown JM, Recht $L$ and Strober S (2017) The promise of targeting macrophages in cancer therapy. Clin Cancer Res 23, 3241-3250

6. Scodeller P, Simón-Gracia L, Kopanchuk S et al (2017) Precision targeting of tumor macrophages with a CD206 binding peptide. Sci Rep 7, 14655

7. Genard G, Lucas S and Michiels C (2017) Reprogramming of tumor-associated macrophages with anticancer therapies: radiotherapy versus chemo- and immunotherapies. Front Immunol 8, 828

8. Pollari M, Brück O, Pellinen T et al (2018) PD-L1 ${ }^{+}$tumorassociated macrophages and PD- $1^{+}$tumor-infiltrating lymphocytes predict survival in primary testicular lymphoma. Haematol 103, 1908-1914

9. Gordon SR, Maute RL, Dulken BW et al (2017) PD-1 expression by tumour-associated macrophages inhibits phagocytosis and tumour immunity. Nature 545, 495-499

10. Mantovani A, Marchesi F, Malesci A, Laghi L and Allavena P (2017) Tumour-associated macrophages as treatment targets in oncology. Nat Rev Clin Oncol 14, 399-416

11. Melotte V, Qu X, Ongenaert M et al (2010) The N-myc downstream regulated gene (NDRG) family: diverse functions, multiple applications. FASEB J 24, 4153-4166

12. Qu X, Zhai Y, Wei H et al (2002) Characterization and expression of three novel differentiation-related genes belong to the human NDRG gene family. Mol Cell Biochem 229, 35-44

13. Park Y, Shon SK, Kim A et al (2007) SOCS1 induced by NDRG2 expression negatively regulates STAT3 activation in breast cancer cells. Biochem Biophys Res Commun 363, 361-367

14. Kim MJ, Lim J, Yang Y, Lee MS and Lim JS (2014) N-myc downstream-regulated gene 2 (NDRG2) suppresses the epithelial-mesenchymal transition (EMT) in breast cancer cells via STAT3/Snail signaling. Cancer Lett 354, 33-42

15. Kim YJ, Kang HB, Yim HS, Kim JH and Kim JW (2013) NDRG2 positively regulates E-cadherin expression and prolongs overall survival in colon cancer patients. Oncol Rep 30, 1890-1898

16. Liang ZL, Kang K, Yoon S et al (2012) NDRG2 is involved in the oncogenic properties of renal cell carcinoma and its loss Is a novel independent poor prognostic factor after nephrectomy. Ann Surg Oncol 19, 2763-2772
17. Li Sj, Wang Wy, Li B et al (2013) Expression of NDRG2 in human lung cancer and its correlation with prognosis. Med Oncol 30, 421

18. Kim B, Nam S, Lim JH and Lim JS (2016) NDRG2 expression decreases tumor-Induced osteoclast differentiation by down-regulating ICAM1 in breast cancer cells. Biomol Ther 24, 9-18

19. Chen $\mathrm{P}$, Zuo H, Xiong $\mathrm{H}$ et al (2017) Gpr132 sensing of lactate mediates tumor-macrophage interplay to promote breast cancer metastasis. Proc Natl Acad Sci U S A 114, 580-585

20. Li H, Huang N, Zhu W et al (2018) Modulation the crosstalk between tumor-associated macrophages and non-small cell lung cancer to inhibit tumor migration and invasion by ginsenoside Rh2. BMC Cancer 18, 579

21. Cassado AdA, D'Império Lima MR and Bortoluci KR (2015) Revisiting mouse peritoneal macrophages: heterogeneity, development, and function. Front Immunol 6, 225

22. Cortés M, Sanchez-Moral L, de Barrios O et al (2017) Tumor-associated macrophages (TAMs) depend on ZEB1 for their cancer-promoting roles. EMBO J 36, 3336-3355

23. Oishi S, Takano R, Tamura S et al (2016) M2 polarization of murine peritoneal macrophages induces regulatory cytokine production and suppresses T-cell proliferation. Immunol $149,320-328$

24. Wang Q, Ni H, Lan L, Wei X, Xiang R and Wang Y (2010) Fra-1 protooncogene regulates IL-6 expression in macrophages and promotes the generation of $\mathrm{M} 2 \mathrm{~d}$ macrophages. Cell Res 20, 701-712

25. Vinnakota K, Zhang Y, Selvanesan BC et al (2017) M2-like macrophages induce colon cancer cell invasion via matrix metalloproteinases. J Cell Physiol 232, 3468-3480

26. Cieslewicz M, Tang J, Yu JL et al (2013) Targeted delivery of proapoptotic peptides to tumor-associated macrophages improves survival. Proc Natl Acad Sci U S A 110, 15919-15924

27. Benner B, Scarberry L, Suarez-Kelly LP et al (2019) Generation of monocytederived tumor-associated macrophages using tumorconditioned media provides a novel method to study tumorassociated macrophages in vitro. J Immunother Cancer 7, 140

28. Choi SC, Kim KD, Kim JT et al (2010) NDRG2 is one of novel intrinsic factors for regulation of IL-10 production in human myeloid cell. Biochem Biophys Res Commun 396, 684-690

29. Salem M, Elbaz O, Zahran M et al (2000) Malignancy: identification of predictors of disease status and progression in patients with myeloma (MM). Hematology 5, 41-45

30. Conze D, Weiss L, Regen PS et al (2001) Autocrine production of interleukin 6 causes multidrug resistance in breast cancer cells. Cancer Res 61, 8851-8858

31. Knüpfer H and Preiß R (2006) Significance of interleukin-6 (IL-6) in breast cancer (review). Breast Cancer Res Treat $102,129-135$

32. Zhang S, Che D, Yang F et al (2017) Tumor-associated macrophages promote tumor metastasis via the TGF- $\beta / S O X 9$ axis in non-small cell lung cancer. Oncotarget 8, 99801-99815

33. Liu L, Ye Y and Zhu X (2019) MMP-9 secreted by tumor associated macrophages promoted gastric cancer metastasis through a PI3KJAKT/Snail pathway. Biomed Pharmacother 117, 109096

34. Zhang X, Goncalves R and Mosser D (2008) The isolation and characterization of murine macrophages. Curr Protoc Immunol Chapter 14: Unit 14.1 